\title{
The optical morphology of A3667 re-examined
}

\author{
M. Johnston-Hollitt ${ }^{1}$, R. W. Hunstead ${ }^{2}$, and E. Corbett ${ }^{3}$ \\ 1 School of Maths \& Physics, University of Tasmania, Private Bag 21, Hobart, Tas 7001, Australia \\ e-mail: Melanie. JohnstonHollitt@utas . edu. au \\ 2 School of Physics, University of Sydney, NSW 2006, Australia \\ 3 Anglo-Australian Observatory, PO Box 296, Epping NSW 1710, Australia
}

Received 23 October 2004 / Accepted 21 November 2007

\section{ABSTRACT}

\begin{abstract}
The galaxy cluster A3667 was observed using the Two-degree Field (2dF) multifibre spectroscopic system on the Anglo-Australian Telescope in a program designed to examine the velocity structure in the region. Specifically, we sought evidence from the optical data for the putative cluster merger believed to be responsible for the observed radio and X-ray emission. We present 184 new redshifts in the region, of which 143 correspond to member galaxies of A3667. We find the cluster velocity distribution to be well modelled by a single Gaussian in agreement with previous results. In addition, new redshift-selected isodensity plots significantly reduce the prominence of the previously reported subgroup to the north-west of the main cluster. Instead, we find the galaxy distribution to be elongated and well mixed, with a high velocity dispersion and no significant evidence for substructure. These results are consistent with the axis of the proposed merger being close to the plane of the sky.
\end{abstract}

Key words. galaxies: clusters: individual: A3667

\section{Introduction}

The ACO galaxy cluster, A3667 (Abell et al. 1989), is a rich, $\mathrm{X}$-ray-luminous cluster, which is arguably one of the most interesting and, consequently, most studied clusters in the southern sky. It has the distinction of being one of only a handful of galaxy clusters to have a cold gas front at the centre (Vikhlinin et al. 2001) and the only cluster yet seen with two bright, diffuse, radio emission regions straddling the $\mathrm{X}$-ray gas (Röttgering et al. 1997). Our physical picture of the cluster is becoming clearer as more multiwavelength data are obtained, but many basic questions about the dynamical history of A3667 remain unanswered.

A3667 is relatively close, with a published mean redshift of 0.055 and a large velocity dispersion $\left(\sigma_{\mathrm{v}}\right)$ of $1000-1500 \mathrm{~km} \mathrm{~s}^{-1}$ depending on the number of galaxies used and the redshift limits applied (Girardi et al. 1998; Melnick \& Quintana 1981; Proust et al. 1988; Sodré et al. 1992). The Abell richness class is 2 and the Bautz-Morgan type is I-II (Abell et al. 1989), indicating a high galaxy density in this region. Proust et al. (1988) presented evidence for a bimodal spatial distribution of galaxies selected by apparent magnitude, and a similar conclusion was reached by Sodré et al. (1992).

ROSAT observations revealed a high X-ray luminosity $L_{\mathrm{X}}$, consistent with the $L_{X}-\sigma$ relation (Xue \& Wu 2000), of $8.74 \times 10^{44} h^{2} \mathrm{erg} \mathrm{s}^{-1}$ in the $0.4-2.4 \mathrm{keV}$ range (Ebeling et al. 1996), making it one of the brightest X-ray sources in the southern sky $^{1}$. Further, the X-ray isophotes are distorted in the direction of the reported bimodal optical distribution and observations from the Einstein satellite are reported as showing clear evidence of substructure (Sodré et al. 1992). Observations of the temperature of the X-ray gas with ASCA by Markevitch et al. (1999) showed the central part of the cluster to be cool and the X-ray

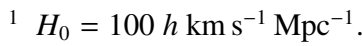

surface brightness profile along the elongation axis suggests a shocked region is present. Recent Chandra observations confirmed this interpretation, revealing the central part of the cluster to contain a cold gas front moving through the warm intracluster medium (Vikhlinin et al. 2001).

Perhaps the most dramatic features of A3667 are the two extended, symmetrically located regions of diffuse radio emission (Röttgering et al. 1997), first detected in $843 \mathrm{MHz}$ images obtained with the Molonglo Observatory Synthesis Telescope, and then confirmed and studied in detail at 1.4 and $2.4 \mathrm{GHz}$ with the Australia Telescope Compact Array, ATCA (Johnston-Hollitt 2003). The combination of these features has been interpreted as evidence that the cluster is observed in a post-merger state after close core passage (Knopp et al. 1996; Markevitch et al. 1999; Roettiger et al. 1999; Röttgering et al. 1997; Vikhlinin et al. 2001).

Given the strong indicator of merger activity present in the radio, X-ray and previous optical data we undertook a set of spectral observation of the objects in the cluster to further test for signatures of a cluster-wide merger in the optical population of A3667.

In Sect. 2 we discuss the signatures of mergers that have been observed in galaxy clusters in the optical and present a detailed summary of the previous optical data collected for A3667. Sections 3 and 4 discuss the Two-degree Field (2dF) observations and data reduction. The major results are discussed in Sect. 5 and the discussion and conclusions are presented in Sect. 6.

\section{Previous optical observations of $A 3667$}

Melnick \& Quintana (1981) obtained redshifts for 16 member galaxies of A3667. Following this, Proust et al. (1988), hereafter 
referred to as P88, obtained a further 31 redshifts for galaxies in the field of A3667, which they combined with the previous results. Unfortunately, this combined sample has since been found to contain some unreliable redshift values (Sodré et al. 1992). P88 also posited a bimodal spatial distribution of galaxies in the field of A3667 based on an isodensity plot of 423 galaxies with $m_{\mathrm{B}} \leq 19$. Two groupings can be seen in this plot. The main group was assigned a redshift of 0.0550 , being that of the galaxy closest to the centre of A3667, while the subgroup to the northwest was suggested to be at $z=0.0563$, based on the redshift of a nearby galaxy. It should be noted that nearly $90 \%$ of the galaxies in this analysis had unknown redshifts and there is an inevitable contribution from foreground and background objects.

Sodré et al. (1992) expanded on the P88 data by measuring redshifts for 128 galaxies in the vicinity of A3667. This sample, hereafter referred to as $\mathrm{S} 92$, included reobservation of all but five of the galaxies in the P88 sample and revealed some large discrepancies in redshift. As neither study published spectra this has made it difficult to determine which was the more reliable; in general the S92 sample has smaller quoted errors and is assumed to be more reliable. Other data in the literature found using a search with the NASA Extragalactic database (NED) have provided a further 28 redshifts for objects in the S92 sample, bringing the total number of redshifts available in the region of A3667 to 161 . Of these, 155 fall in the range $0.044 \leq z \leq 0.068$ and are likely to be cluster members.

Girardi et al. (1996) studied A3667 as part of a sample of 37 galaxy clusters used to examine the relationship between velocity dispersion, $\sigma_{\mathrm{v}}$, and X-ray temperature, $T$. They argued that since both $\sigma_{\mathrm{v}}$ and $T$ were related to a cluster's gravitational potential there would only be a strong correlation between these quantities for clusters in which both gas and galaxies were in dynamical equilibrium with the cluster potential. Using the 123 redshifts from the S92 sample, Girardi et al. (1996) examined A3667 for substructure using both a velocity distribution and velocity gradient analysis. They found neither significant multiple peaks in the velocity distribution, nor a velocity gradient. Using the method of Dressler \& Shectman (1988) they computed the probability of substructure as 0.697 where a result above 0.990 was considered significant. Despite the lack of statistical evidence, their isodensity plot was reminiscent of the P88 plot and suggested some substructure to the northwest of the cluster centre. The velocity dispersion was determined to be $1208_{-84}^{+95} \mathrm{~km} \mathrm{~s}^{-1}$.

Expanding on their earlier work, Girardi et al. (1998) used 154 of the then known 155 redshifts for A3667 to compute a maximum cluster radius of $2.22 h^{-1} \mathrm{Mpc}$, a mean galactocentric redshift of 0.0566 and a global velocity dispersion of $971_{-47}^{+62} \mathrm{~km} \mathrm{~s}^{-1}$. Girardi et al. (1998) also obtained a virialised radius of $1.94 h^{-1} \mathrm{Mpc}$ in which 152 of the galaxies with previously measured redshifts are contained. The total virialized mass was calculated to be $15.98_{-1.71}^{+2.18} \times 10^{14} M_{\odot}$. They further concluded that the velocity distribution was isotropic. In comparison Knopp et al. (1996) give the X-ray radius of the cluster to be $R_{\mathrm{X}}=0.80 h^{-1} \mathrm{Mpc}$ and the X-ray mass at $M_{\mathrm{X}}=$ $3.50_{-0.40}^{+0.30} \times 10^{14} M_{\odot}$. Girardi et al. (1998) obtain an optical mass of $M_{\mathrm{O}}=4.89_{-0.52}^{+0.67} \times 10^{14} M_{\odot}$ within this X-ray radius. Assuming that the cluster galaxies follow the gravitational potential, the good agreement between these mass estimates strongly suggests that the cluster is in dynamical equilibrium (Girardi et al. 1998).

The mean redshifts and velocity dispersions obtained in the studies cited above are summarized in Table 1.
Table 1. Mean redshifts and velocity dispersions for A3667.

\begin{tabular}{lcll}
\hline \hline Reference & $N$ & Mean $z$ & $\sigma_{\mathrm{v}}\left(\mathrm{km} \mathrm{s}^{-1}\right)$ \\
\hline Proust et al. (1988) & $46^{*}$ & $0.054 \pm 0.003$ & $1606 \pm 288$ \\
Sodré et al. (1992) & 120 & $0.0553 \pm 0.0004$ & $1197 \pm 79$ \\
Girardi et al. (1996) & 123 & & $1208_{-84}^{+95}$ \\
Girardi et al. (1998) & 154 & 0.0566 & $971_{-47}^{+62}$ \\
This paper & 231 & $0.0555 \pm 0.0003^{\ddagger}$ & $1102^{ \pm} \pm 6$ \\
\hline
\end{tabular}

${ }^{*}$ From the table in Proust et al. (1988). ${ }^{\ddagger}$ The mean of all 231 available redshifts; the best-fit Gaussian to the data gives $\bar{z}=0.0553$ (see Sect. 6).

\section{2dF observations}

In order to increase the redshift sample and examine the distribution of galaxy types in the cluster, observations were undertaken with the $2 \mathrm{dF}$ spectrographic system (Bailey et al. 2002) on the AAT. SuperCOSMOS plate scans were used to obtain a list of suitable galaxies with $15.5 \leq m_{\mathrm{B}} \leq 18.3$. As there is a marked discrepancy between the redshift values for some objects observed by P88 and S92 we decided to include a subset of the S92 catalogue in the target sample to check for any systematic differences in redshift. The optical counterparts of the known radio galaxies in the region (Johnston-Hollitt 2003) were also selected. This produced a final configuration of 338 target objects, with a further 49 fibres assigned to guide stars and sky.

The observations were scheduled on 2001 July 20, using the $270 \mathrm{R}$ and $316 \mathrm{R}$ gratings. A total integration time of $1.5 \mathrm{~h}$ was obtained in three 30-minute integrations to allow cosmic ray removal via a sigma-clipping algorithm. This gave a wavelength coverage of approximately $3600-8000 \AA$, varying slightly between the two spectrographs due to the slightly different dispersions of the gratings and from fibre to fibre on each spectrograph. The instrumental resolution was $9.9 \AA$ FWHM.

\section{Data reduction}

Data were reduced using the $2 \mathrm{dfdr}$ pipeline software package, which was developed for the $2 \mathrm{dF}$ Galaxy Redshift Survey (Colless 1998; Colless et al. 2001). A full description of the semi-automated extraction and reduction procedure is given in these references.

The software performs two independent redshift estimations depending on the emission and absorption properties of the spectra. For the absorption spectra we used the standard technique of cross-correlation (Tonry \& Davis 1979) with a set of eight absorption templates, five galaxies and three stars. The galaxies used and their morphological types, as listed in Colless et al. (2001), are: (1) NGC 3379 (E), (2) NGC 4889 (cD), (3) NGC 5248 (Sbc), (4) NGC 2276 (Sc) and (5) NGC 4485 $(\mathrm{Sm} / \mathrm{Im})$. The stars used and their spectral classes are (6) HD 116608 (A1V), (7) HD 23524 (K0V) and (8) BD05 1668 (M5V).

Spectra are prepared for cross-correlation via a six-step process that includes: continuum-subtraction; clipping strong emission lines; rebinning to a logarithmic scale; apodization; Fourier transformation; and application of an exponential filter. Further details of each process can be found in Colless et al. (2001).

The highest peak in the cross-correlation function is then fitted with a quadratic equation to obtain its position and height. The ratio of the peak height to the noise level in the crosscorrelation function is subsequently computed and used to determine the quality of the redshift estimate, via the assignment 
of a quality flag, $Q_{\mathrm{a}}$, to each spectrum. The value of $Q_{\mathrm{a}}$ ranges from 1 to 4 , with 4 and 3 meaning a reliable fit, 2 a probable fit and 1 an unreliable fit. If no fit is obtained a quality factor of 0 is assigned. When $Q_{\mathrm{a}}$ is 3 or 4 , there is an additional requirement that at least four or six of the eight templates, respectively, produce the same redshift to within $600 \mathrm{~km} \mathrm{~s}^{-1}$. In addition, the code also outputs which of the eight templates produced the best correlation with each spectrum; this gives information on the object's morphological type.

For emission spectra the code performs a Gaussian profile fit to each line after first subtracting the continuum. Any peaks in the resultant spectrum which are higher than 3.3 times the RMS noise are marked as candidate emission lines. The three strongest lines in each spectrum are then tested in pairs for line separations consistent with a known redshift from a set of common emission lines ([O II], $\mathrm{H} \beta$, [O III], $\mathrm{H} \alpha$ or [N II]). If a match is found then all other emission lines that match that redshift to within $600 \mathrm{~km} \mathrm{~s}^{-1}$ are found, and the mean redshift is adopted as the emission redshift. If no two emission lines are found to match a particular redshift for a pair of common lines, (or there are fewer than three lines in total) then up to two single-line redshifts are retained for comparison with the absorption derived redshift. A quality flag for this redshift estimate, $Q_{\mathrm{e}}$, is assigned based on the number of features fitted. This number can take the values $0,1,2,4$ with 0 being no lines, 1 for one line, 2 for two lines and 4 for three or more.

The code then compares the redshift obtained via each method and selects the one with the higher quality flag. In the case where $Q_{\mathrm{a}}=Q_{\mathrm{e}}$ the absorption value is selected. The best quality flag, $Q_{\mathrm{b}}$ is then given as the greater of $Q_{\mathrm{a}}$ or $Q_{\mathrm{e}}$, with two exceptions: (1) if the difference in redshifts derived via the two methods is less than $600 \mathrm{~km} \mathrm{~s}^{-1}$ then $Q_{\mathrm{b}}$ is the greatest of $Q_{\mathrm{a}}$, $Q_{\mathrm{e}}$ or 3 and (2) if $Q_{\mathrm{a}}$ and $Q_{\mathrm{e}}$ are both less than 2 and the difference in redshifts derived via the two methods is greater than $600 \mathrm{~km} \mathrm{~s}^{-1}$ then $Q_{\mathrm{b}}$ is set to 1 .

After the automatic quality flags are assigned the spectra are then inspected manually and interactively re-fitted if necessary. Also at this time the observer may assign their own quality flag $Q$ (independent of the automatic quality flag $Q_{\mathrm{b}}$ ) on a scale of 1 to 5 , with 5 corresponding to spectra of the highest quality.

\section{Results}

Of the 338 spectra obtained, 136 turned out to be misclassified stars. This was later tracked down to a problem with the object discriminator software for bright objects in the pre-release SuperCOSMOS data. For target selection we set the lower magnitude limit to $m_{\mathrm{B}}=15.5$, at the bright end of the magnitude distribution of cluster members. Unfortunately, this was too bright for the object classification software to distinguish reliably between stars and galaxies.

Figure 1 shows the distribution of redshifts obtained from all object fibres, with bin intervals of $\Delta z=0.002$. This figure has two main features; the misclassified stars near zero redshift and A3667 members at around $z=0.056$. The inset A3667 histogram shows remarkably little velocity structure either surrounding the main cluster group or within it. There is no evidence of distinct in-falling groups or other velocity features around the main cluster as found in other merging systems like the A3125/A3128 complex (Rose et al. 2002). Then again, perhaps this is not surprising as clusters which harbour diffuse radio emission have been shown to be statistically more isolated than clusters of similar X-ray luminosity (Schuecker \& Böhringer 1999).

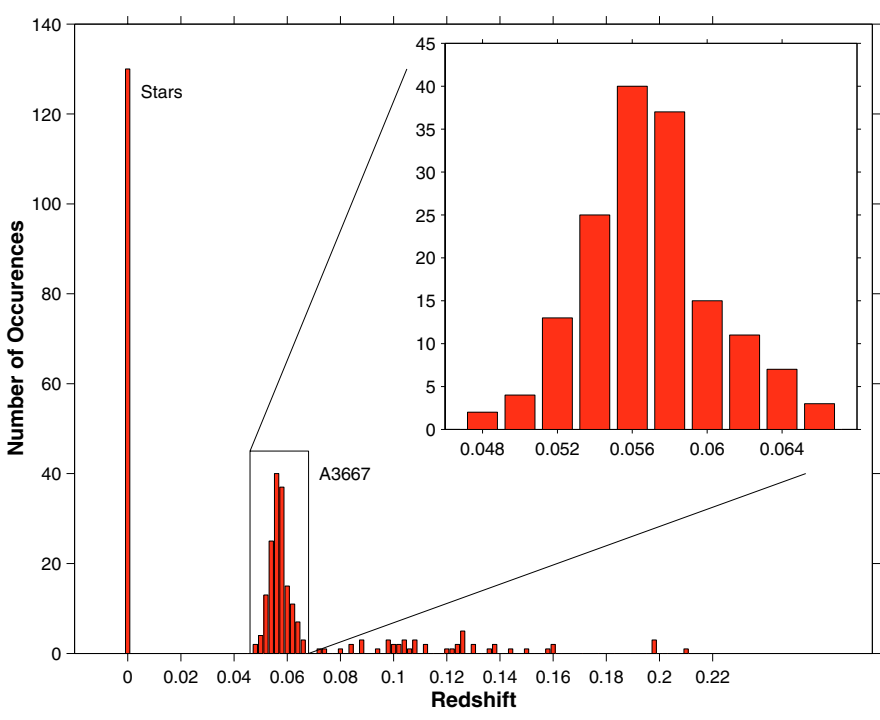

Fig. 1. 2dF redshift histogram for the A3667 field; the inset plot shows an enlarged version of the cluster redshift distribution.

After the stars had been removed, the remaining 202 spectra were examined individually for quality of fit. Spectra for which the quality flag $Q_{\mathrm{b}}$ was less than 2 were treated with caution and 18 spectra with low signal-to-noise were removed from the sample. Of the remaining 184 spectra, 143 fell within the range $z=0.044-0.068$ and are considered to be members of A3667. This redshift-restricted sample of 143 galaxies is published online at CDS in the format of Table 2; a sky plot of this sample, together with the 154 values from the literature, is shown in Fig. 2. The remaining 41 galaxy spectra not related to A3667 but obtained during these observations are also published online at CDS in a format similar to those in Table 2.

There are 67 galaxies in common between the $2 \mathrm{dF}$ and literature samples. As can be seen from Fig. 3, there is generally good redshift agreement between the two datasets. Analysis of the differences in redshift gives a median difference of zero and a standard deviation of $\Delta z=0.00036\left(102 \mathrm{~km} \mathrm{~s}^{-1}\right)$, well below the combined redshift uncertainty $\left(\sim 200 \mathrm{~km} \mathrm{~s}^{-1}\right)$. This suggests that it is acceptable to combine the two datasets.

In merging the two datasets we took note of the $2 \mathrm{dF}$ quality parameters. The blunder rate for spectra measured in the 2dFGRS for which $2 \mathrm{dfdr}$ has assigned $Q_{\mathrm{b}}>2$, is only $1.6 \%$ and this depends strongly on magnitude, with almost all blunders occurring for $m_{\mathrm{B}} \geq 18$ (Colless et al. 2001). Thus, for a comparatively bright sample such as this, one can have confidence in the reliability of the $2 \mathrm{dF}$ redshifts. When combining the two datasets for subsequent analysis the $2 \mathrm{dF}$ redshifts were used in preference to the literature values in all cases where $Q_{\mathrm{b}}$ was greater than two. Of the nine $2 \mathrm{dF}$ spectra with $Q_{\mathrm{b}} \leq 2$, seven agreed well with the previously published redshifts and were preferred for the combined dataset. The remaining two spectra showed large discrepancies of $\Delta z=0.0069$ and 0.0027 (corresponding to $\sim 2000$ and $800 \mathrm{~km} \mathrm{~s}^{-1}$ respectively). These points can be seen in Fig. 3 as outliers numbered 105 and 78 (which correspond to their identification numbers in Table 2). Both objects were quite bright with magnitudes of 16.90 and 17.39, and quoted errors of 61 and $200 \mathrm{~km} \mathrm{~s}^{-1}$ respectively (Sodré et al. 1992); for consistency, the $2 \mathrm{dF}$ values were retained for analysing the redshift distribution. The remaining two labelled outliers, 70 and 101 , were assigned the highest quality flags by $2 \mathrm{dfdr}$ and were thus assumed to be correct. This gave a combined dataset of 
Table 2. Redshifts for the first 10 galaxies* in the sample of 143 measured with $2 \mathrm{dF}$ in the cluster A3667 and falling in the redshift range $0.044 \leq z \leq 0.068$.

\begin{tabular}{ccccccccc}
\hline \hline ID & $\begin{array}{c}\text { RA } \\
(\mathrm{J} 2000)\end{array}$ & $\begin{array}{c}\text { Dec } \\
(\mathrm{J} 2000)\end{array}$ & $m_{\mathrm{B}}$ & $z_{\text {abs }}$ & $z_{\mathrm{em}}$ & $z_{\text {ave }}$ & $z_{\text {lit }}$ & $\begin{array}{c}\text { morphological } \\
\text { type }\end{array}$ \\
\hline 1 & 200537.49 & -564106.1 & 16.89 & 0.0558 & & & & 2 \\
2 & 200626.98 & -561950.9 & 18.16 & 0.0586 & & & & 3 \\
3 & 200722.03 & -563944.0 & 16.41 & 0.0535 & & & & 5 \\
4 & 200729.09 & -572846.2 & 18.09 & 0.0553 & 0.0553 & 0.0553 & & 3 \\
5 & 200730.08 & -572258.6 & 16.75 & 0.0521 & 0.0518 & 0.0520 & & 4 \\
6 & 200732.50 & -563445.9 & 17.43 & 0.0560 & 0.0557 & 0.0559 & & 5 \\
7 & 200756.06 & -561601.3 & 16.65 & 0.0543 & 0.0545 & 0.0544 & 0.0535 & 3 \\
8 & 200801.56 & -560607.6 & 17.60 & 0.0594 & 0.0594 & 0.0594 & & 2 \\
9 & 200809.17 & -563021.7 & 16.80 & 0.0523 & & & 0.0524 & 3 \\
10 & 200827.61 & -562042.5 & 17.79 & 0.0572 & 0.0569 & 0.05705 & & \\
\hline
\end{tabular}

* The full data table contains 143 objects and is available in electronic form at CDS via anonymous ftp to cdsarc.u-strasbg.fr (130.79.128.5) or via http://cdsweb.u-strasbg.fr/cgi-bin/qcat?J/A+A/479/1. Column 1 gives the source ID number; Cols. 2 and 3 are the Right Ascension and Declination in J2000 coordinates; Col. 4 is the blue magnitude; Cols. 6 and 7 are the redshifts as determined by absorption and emission features, respectively, and Col. 8 is the average redshift for cases where a redshift could be determined by both emission and absoprtion features; Col. 9 is the previously published redshift and Col. 10 is the morphological type determined by the best correlation to the spectral templates used by $2 \mathrm{dfdr}$, numbers correspond to those listed in Colless et al. (2001).

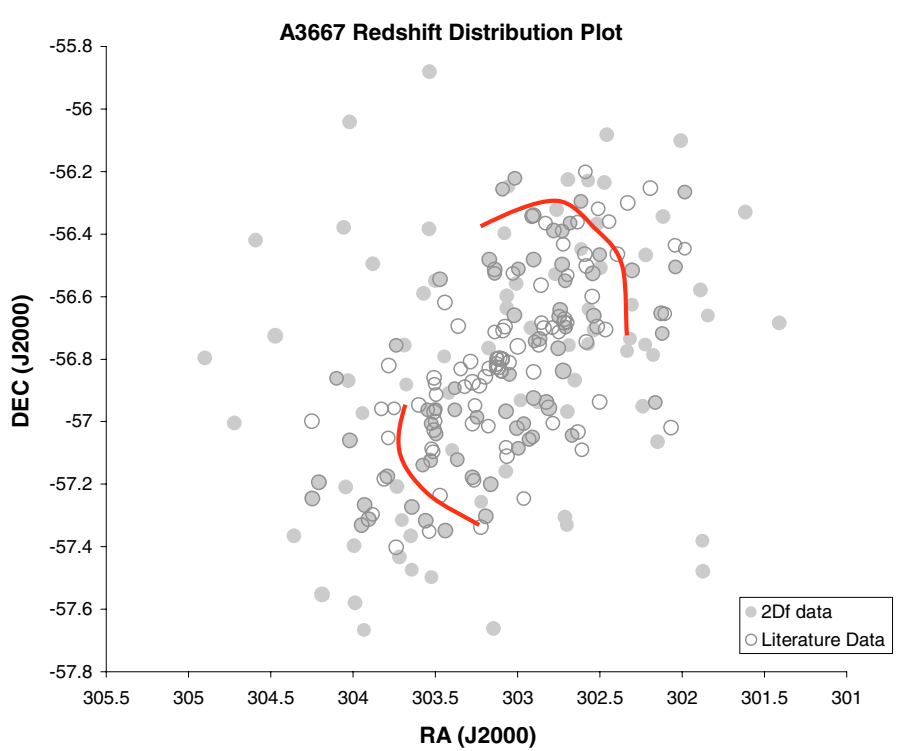

Fig. 2. Distribution on the sky of galaxies with measured redshifts in the region of A3667. Filled circles are the $2 \mathrm{dF}$ data and open circles are the redshifts from the literature. Where the $2 \mathrm{dF}$ and literature datasets agree in both position and redshift, the circle is shown filled and outlined. The arcs denote the outside edges of the radio relics.

231 redshifts in the range 0.044 to 0.068 , which were then used in the subsequent analysis.

It should be noted that these observations combined with those previously published mean that A3667 has been spectroscopically observed at a completeness level of $70 \%$ down to our limiting magnitude of $b_{j}=18.3$. Figure 4 shows all galaxies in a field of one degree radius centred on the peak in X-ray emission down to $b_{j}=18.3$ as gray points overlaid with all galaxies for which spectroscopic redshifts are currently available.

\subsection{Redshift distribution}

The redshift distribution for the combined dataset is shown in Fig. 5, overlaid with a Gaussian fit to the central peak at $z=0.0555$. While the redshift distribution is well modelled by a Gaussian, there is some evidence for a small subgroup of

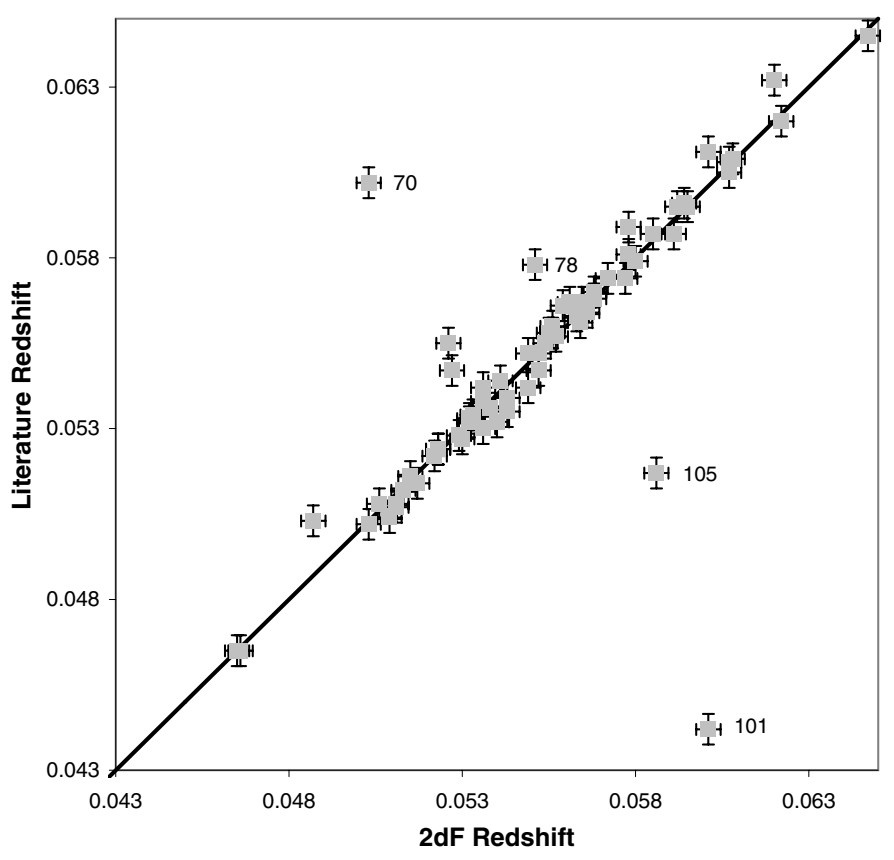

Fig. 3. Redshift comparison for A3667. The literature redshift is plotted against the $2 \mathrm{dF}$ redshift for the 67 sources in common. Clustering about the slope of unity suggests there is no overall systematic difference between the two datasets. The four labelled outliers are discussed in the text; the number corresponds to its identification number in the online version of Table 2 .

galaxies at a slightly higher redshift; however, the galaxies belonging to this possible subgroup are not spatially localised but are spread across the cluster. To search for possible subgroups a pseudo-3D plot of RA, Dec and redshift was constructed using Matlab to explore the spatial distribution of all 231 galaxies. While such a plot does not give a physical 3D model of the cluster, it is a useful tool in searching for associations. Standard redshift versus $\triangle \mathrm{RA}$ and $\triangle \mathrm{Dec}$ plots were also made (see Fig. 6) and tested for associations, on the premise that if subgroups are present there will be tight associations between the member galaxies in both plots. No likely subgroups were found via visual inspection and the cluster appears extremely well mixed. 


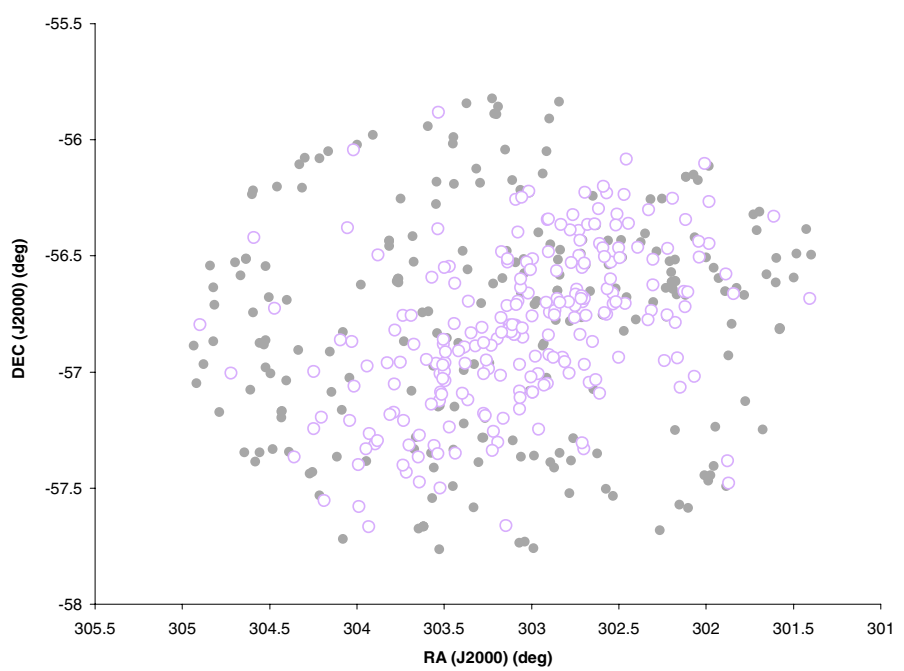

Fig. 4. Coverage of galaxies for which spectroscopic redshifts have been determined. Filled gray circles are all the galaxies down to $b_{j} \leq 18.3$ in a one degree radius about A3667 as determined from SuperCOSMOS plate scans while open circles represent those galaxies for which redshifts have been obtained. The average completeness over the field is $70 \%$.

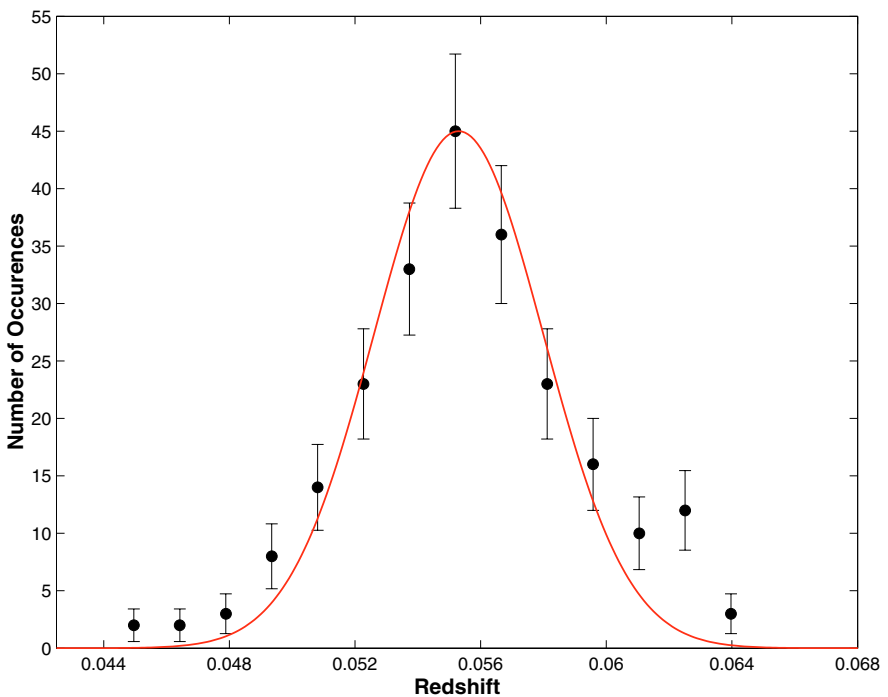

Fig. 5. Redshift distribution for A3667 with a Gaussian fitted to the central peak. The Gaussian is centred at the mean redshift of $z=0.0555$ and there is a weak excess at around 0.0625 . The velocity dispersion is $1102 \mathrm{~km} \mathrm{~s}^{-1}$.

This lack of velocity structure is consistent with previous results (Girardi et al. 1996).

Statistical analysis of the total dataset gives a mean redshift of 0.0555 , and a velocity dispersion, $\sigma_{\mathrm{v}}$, of $1102 \pm 46 \mathrm{~km} \mathrm{~s}^{-1}$, which is slightly higher than the previously published values.

\subsection{Quantitative tests for substructure}

In order to produce a more quantitative analysis of the likely substructure present we performed 2 and 3-dimensional Lee-Fitchett tests (Fitchett 1988; Fitchett \& Webster 1987) in addition to calculating the $\Delta$ statistic for the cluster (Dressler \& Shectman 1988).
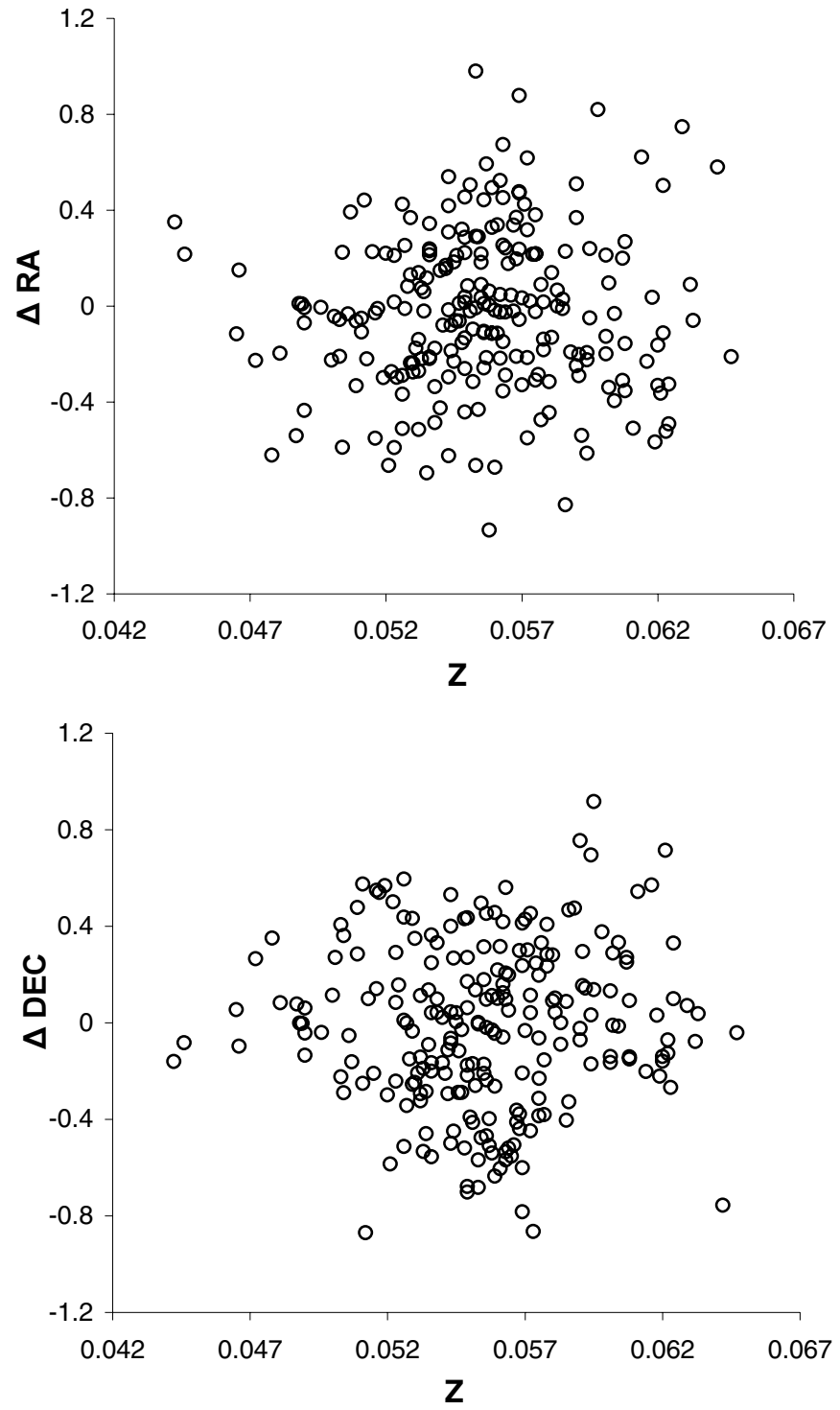

Fig. 6. Redshift distribution for the combined dataset of 231 galaxies as a function of RA and Dec, measured in degrees from the cluster centre.

\subsubsection{Lee-Fitchett test}

We performed a Lee-Fitchett (LF) test (Fitchett 1988; Fitchett $\&$ Webster 1987) on the 231 galaxies known to be members of A3667. The ratio obtained was 5.15 which is highly significant. However, as the LF test is simply a measure of spatial variations in variance, this does not imply subclustering in the case of A3667 which is highly aspherical. Although the Lee-Fitchett test has been shown to be a good indicator of substructure it does have limitations in that it will breakdown in the case of two widely spatially separated Gaussian velocity distributions. In the case of A3667 the LF test returns a result for significant substructure only in the plane of the sky along the axis of elongation of the galaxy positions. This is to be expected given that the premise of the test is that clusters are roughly spherical. In order to assess the veracity of the test we simulated several clusters of the same size as A3667 with a high degree of aspherity (elliptically distributed in RA-Dec) but which have a Gaussian velocity distribution. In all cases the simulated clusters also returned a highly significant substructure value for the LF test along the major axis of the ellipse defining the galaxy positions in RA 
Table 3. KMM bimodal fitting results for the A3667 redshift sample.

\begin{tabular}{lcccc} 
& \multicolumn{2}{c}{ Input parameters } & \multicolumn{2}{c}{ Output parameters } \\
\cline { 2 - 5 } & Group1 & Group2 & Group1 & Group2 \\
\hline Mean Group $z$ & 0.0555 & 0.0625 & 0.0555 & - \\
Proportion & 0.935 & 0.065 & 1.0 & 0 \\
No. of Galaxies & 216 & 15 & 231 & 0 \\
\hline
\end{tabular}

and Dec. Neither in the case of A3667 or the simulated clusters was there any significant axis of substructure associated with the velocity data. Thus, all we can say from this test is that the distribution of galaxies in A3667 is elongated in the plane of the sky, as seen readily from the RA-Dec plots.

\subsection{2. $\Delta$ statistic}

We undertook the calculation of the $\Delta$ statistic (Dressler \& Shectman 1988), which tests whether the distribution of radial velocities $(\bar{v}, \sigma)$ of a local $(N=15)$ population of galaxies changes with position across the cluster. The $\Delta$ value returned for A3667 was 1.26. For comparison 100000 Monte-Carlo simulations, in which the positions of the galaxies were fixed in RA and Dec but randomly shuffled in radial velocity, returned a distribution of $\Delta$ values ranging from 0.76 to 1.49 with a mean of 1.09 and standard deviation of 0.10 . The lack of a clear difference from the $\Delta$ value determined for A3667 suggests that there is no overall correlation between position and redshift in these data and, therefore, no significant substructure.

\subsection{Bimodality testing}

A series of tests were performed to pursue the issue of bimodality in the cluster raised by P88 and S92.

\subsubsection{Velocity gradient}

If the bimodal distribution reported by P88 is real and the two subgroups are at all inclined from the plane of the sky, we would expect to observe a gradient in radial velocity across the cluster. The Matlab cube referred to in Sect. 5.1 was used to search for a velocity gradient across the cluster; none was found.

\subsubsection{Kaye's mixture model}

To test quantitatively whether a single Gaussian is an adequate fit to the redshift distribution shown in Fig. 5, we used Kaye's Mixture Model algorithm, KMM (Ashman et al. 1994), which allows a user-specified number of Gaussian profiles to be fitted to the unbinned, raw data and provides a statistical comparison with a single Gaussian fit. The user provides an estimate of the mean value of each peak and the fraction of the members in each peak. The software, provided by Keith Ashman, then assigns membership of each redshift to one of the initially supplied Gaussian profiles, and returns posterior probabilities of group membership for each profile.

The algorithm was applied for the two-profile case and Table 3 summarises the input and output parameters. For the 231 redshifts measured for A3667, the code assigned all values to be members of the main group centred about $z=0.0555$, with an overall confidence level of $96.7 \%$.
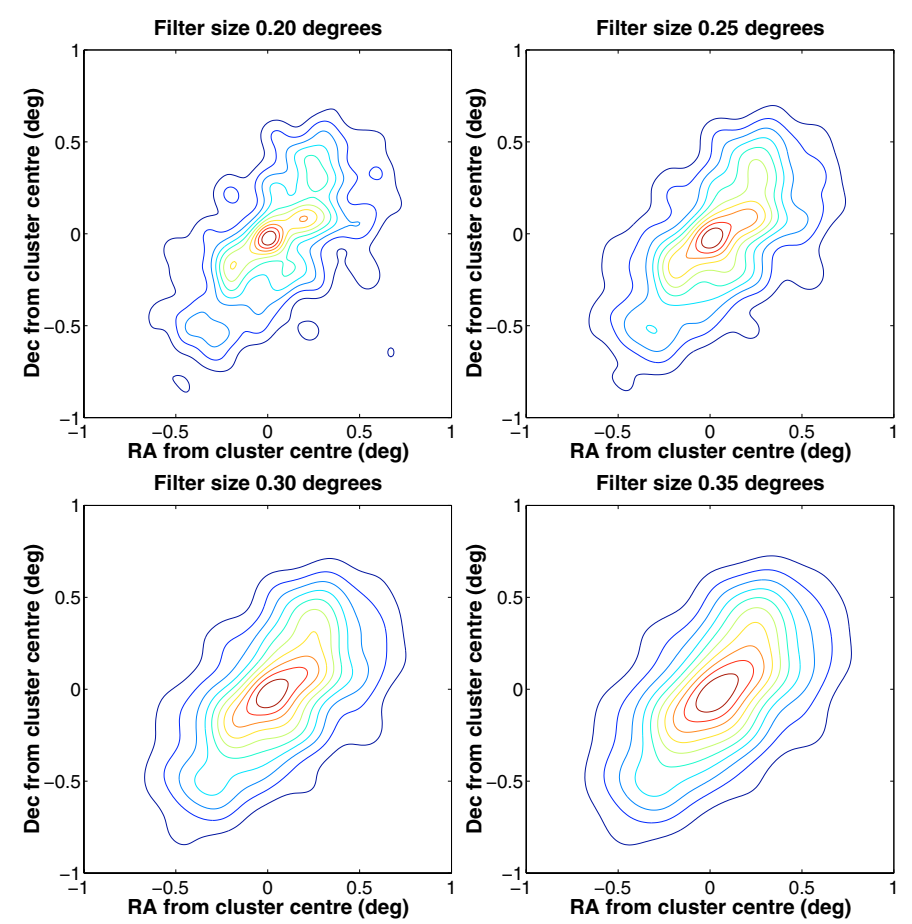

Fig. 7. Galaxy isodensity plots for A3667, generated by application of Gaussian filters of various sizes (from $\sigma=0.2$ to $0.35^{\circ}$ ) to the 231 known cluster members. Contours are shown at $\sqrt{2}$ intervals.

\subsubsection{Kurtosis and skewness}

Tests for kurtosis and skewness were applied to the velocity data. The kurtosis returned a value of 0.233 which is within 1 Standard Error of Kurtosis (SEK) for a Gaussian distribution with the same number of points $(\mathrm{SEK}=0.3223)$. The skewness of the velocity distribution was found to be 0.134 which again was within 1 Standard Error of Skewness (SES) for a Gaussian distribution with 231 values $(\mathrm{SES}=0.16$ ). For skewness to be considered significant we would expect values approaching two. Thus there appears to be no significant skewness or kurtosis for the velocity distribution as compared with a Gaussian distribution of the same number of data points.

\subsubsection{Isodensity plots}

In order to assess the bimodality of the galaxy surface distribution, Gaussian smoothing was applied to the spatial distribution of the 231 galaxies with redshifts between 0.044 and 0.068 . Figure 7 shows the galaxy location data smoothed with Gaussian filters ranging in size from $\sigma=0.20^{\circ}$ to $0.35^{\circ}$. The $0.20^{\circ}$ filter image shows an excellent overall match to the published isodensity plot of P88, except that the redshift-restricted data do not exhibit the significant subgroup structure seen by P88. However, there is a definite elongation of the cluster and some evidence of a slight enhancement in galaxy density in the NW region where P88 find a significant subgroup. There is also a feature of equal significance on the SE side of A3667. This second feature is in the approximate location that Vikhlinin \& Markevitch (2002) predict a small subgroup based on their Chandra analysis of the X-ray features. However, it is likely that both these features result from the limited sample size rather than comprising distinct subgroups. Neither feature persists if the filter size is increased even marginally. 


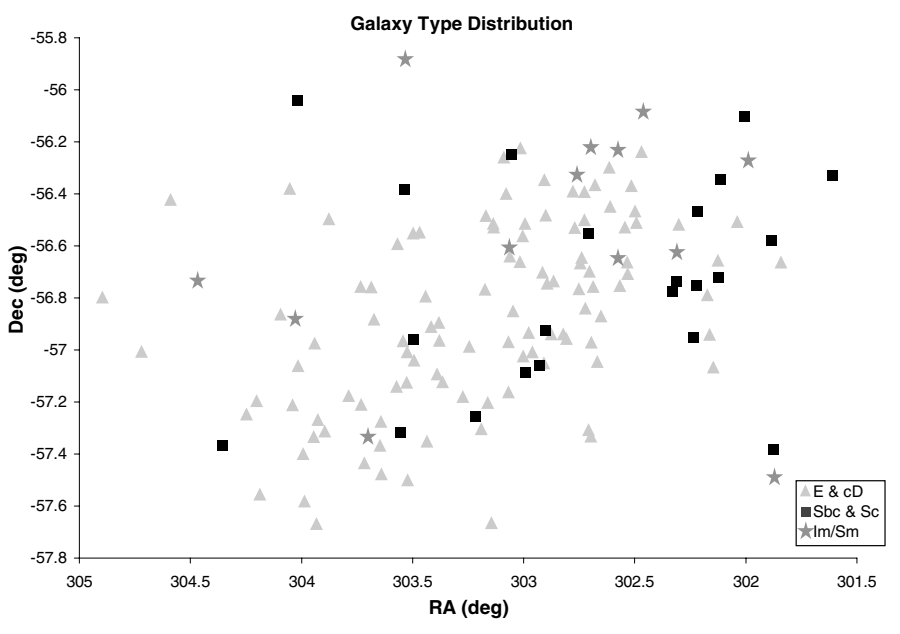

Fig. 8. Galaxy type spatial distribution for the 143 galaxies in the $2 \mathrm{dF}$ dataset.

\subsection{Galaxy morphologies}

As mentioned above, one of the products of the $2 \mathrm{dfdr}$ crosscorrelation redshift determination is the template which gives the strongest correlation with each measured spectrum. This provides information on each galaxy's morphological type. Examination of the spatial distribution of various galaxies within the cluster may provide valuable information on the cluster's dynamical history.

Figure 8 shows the distribution of galaxy types in A3667 as defined by the $2 \mathrm{dfdr}$ template. The morphological types are elliptical (E), central dominant (cD), spiral (Sc, Sbc) and irregular/small (IM/SM). Not surprisingly the predominant galaxy type is elliptical. The spiral population occurs mainly in the north-western part of the cluster and at first glance this appears to be significant. We applied a bi-dimensional KolmogorovSmirnoff test (Peacock 1983) to test if there was a statistically significant difference between the distribution of spiral and elliptical galaxies in the cluster. Using positions of the 85 elliptical galaxies and 19 spirals with morphological types identified in the $2 \mathrm{dF}$ observations we computed a $P\left(>Z_{\infty}\right)$ of 0.999 which supports the null hypothesis that the distributions are drawn from the same population. In addition we calculated the mean redshift and velocity dispersion for the separate populations finding them to be almost identical with $\bar{z}_{\text {elliptical }}=0.05570$ and $\bar{z}_{\text {spiral }}=0.05572$ while $\sigma_{\text {elliptical }}=1007 \pm 109 \mathrm{~km} \mathrm{~s}^{-1}$ and $\sigma_{\text {spiral }}=1133 \pm 260 \mathrm{~km} \mathrm{~s}^{-1}$.

Observations of starburst galaxies have shown a strong correlation between star formation and tidal disruption. Such disruption may be either the result of galaxy mergers, such as the famous case of the Antennae galaxies (NGC 4038 and NGC 4039) (Whitmore et al. 1999), or from accretion or merger shocks in the intra-cluster medium (ICM) (Burns et al. 2002). An excess of starburst galaxies would seem then to indicate a turbulent state, either through more individual galaxy collisions as in the case of CL 1358+62 (Bartholomew et al. 2001), or through shockwave disruption throughout the ICM, possibly resulting in ram pressure stripping. Thus, one might expect that an overdensity of starburst galaxies would be evidence for a recent cluster merger. However, during the late stages of cluster evolution we know from the Butcher-Oemler effect that cluster constituent galaxies are more likely to be gas-stripped red ellipticals than gasrich blue spirals. Without an abundance of gas it is unlikely then that starbursts per se would be observed. A more likely indicator of recent merging might be an excess of the socalled "E+A" galaxies, which represent a post-starburst population (Couch \& Sharples 1987) generated either through galaxygalaxy mergers or ram pressure stripping (Belloni et al. 1995). Several studies have addressed the prevalence of post-starburst galaxies in cluster environments (Hogg et al. 2006; Poggianti 2003; Tran et al. 2007) as well as the field (Blake et al. 2004) finding that the fraction of post-starburst galaxies is different to the field at low redshifts $(z \leq 0.1)$. As an excess of post-starburst galaxies may be an indicator of quenching of star formation in galaxies as a result of the cluster environment (Poggianti 2003) they can be considered an important indicator or merger activity.

No E+A galaxies (Couch \& Sharples 1987) were found among the spectra. Perhaps this is not surprising as only 37 examples with strong $\mathrm{H} \beta, \mathrm{H} \gamma$ and $\mathrm{H} \delta$ lines were found in a subset of over 15000 low- to mid-redshift galaxies observed in the $2 \mathrm{dFGRS}$. This is an incidence rate of only 0.25 percent (Deeley 2000, private communication). While at moderate to high redshifts $\mathrm{E}+\mathrm{A}$ galaxies would be more likely to be found in regions where galaxy-galaxy interactions are prominent, i.e., the central cluster region (Tran et al. 2003) at redshifts less than one they tend to be found in the field (Blake et al. 2004) and only a few have to date been seen in low redshift clusters (Caldwell \& Rose 1997; Galaz 2000) like A3667. Due to the spacing limitations imposed by the physical size of the $2 \mathrm{dF}$ fibre buttons the central region of $\mathrm{A} 3667$ is undersampled by $2 \mathrm{dF}$, as can be seen in Fig. 2 meaning that if there were E+A galaxies in the cluster we would potentially miss them.

\section{Discussion and conclusions}

Cluster mergers are rarely established based solely on evidence from one wavelength regime, generally requiring a multiwavelength approach to reveal the true dynamical nature of the interactions.

The presence of diffuse radio emission in clusters of galaxies points to the existence of cluster-wide magnetic fields of the order of $0.1-1 \mu \mathrm{G}$ and a population of relativistic electrons with Lorentz factor, $\gamma \gg 1000$ and energy densities of $10^{-14}-10^{-13} \mathrm{erg} \mathrm{cm}^{-3}$ (Feretti 2004). Diffuse radio emission is observed most commonly in clusters which are believed to have undergone a recent merger event and this leads naturally to the conclusion that the phenomenon is due to turbulence and shocks in the intra-cluster medium energizing the electron population (Giovannini \& Feretti 2002; Harris et al. 1980; Tribble 1993). Simulations of the turbulence and shocks in the cluster environment are able to predict the characteristics of observed diffuse radio emission (Miniati et al. 2001; Pfrommer et al. 2007; Roettiger et al. 1999) and studies of the number counts suggest that the most likely lifetimes of diffuse radio emission is of the order of 1 Gyr (Kuo et al. 2004). Additionally, theoretical models of merging systems suggest that X-ray substructure will be erased after the sound crossing time and thus, clusters with disturbed X-ray isophotes are likely to be dynamically young and in on-going merger events. Finally, the strongest evidence for clusters being in recent dynamical interactions is the detection of temperature changes across the source (Govoni et al. 2004; Pratt et al. 2007).

While the number of clusters containing diffuse emission is still comparatively low (Feretti 2004), there are now a number of well studied systems with multiwavelength data 
including A2256 (Clarke \& Ensslin 2006), A754 (Henry et al. 2004), IC 1262 (Hudson et al. 2004) as well as the numerous examples presented by Govoni et al. (2004). To date most analysis tends to concentrate on the radio and X-ray properties of the clusters. However, often this is insufficient to determine the merger geometry. For example, the case of A3921 (Ferrari et al. 2006) required data from optical, radio and X-ray analysis to determine the dynamical state of the cluster. In this instance optical observations alone were inconclusive but taken together with the other wavelength data the merger axis could be established. Similarly, high resolution X-ray from XMM-Newton analyzed in conjunction with existing multiwavelength data has proved vital to unravelling the cluster dynamics in several cases such as A85 (Durret et al. 2005) and A3921 (Belsole et al. 2005).

A3667 has a mean redshift of 0.0555 , a high velocity dispersion $\left(1102 \pm 46 \mathrm{~km} \mathrm{~s}^{-1}\right)$ and an elongated optical axis. It is typical of rich clusters in that it is dominated by elliptical galaxies. There is marginal evidence for a grouping of spirals in the northwestern part of the cluster. The velocity distribution is well modelled by a single Gaussian peaking at $z=0.0555$, with a weak excess around a redshift of 0.0625 . There seems to be little or no support for distinct in-falling groups either from the velocity information or the spatial distribution of galaxies with measured redshifts. Moreover, there is no evidence for a velocity gradient across the cluster suggested by the previously reported bimodal optical distribution. However, A3667 is a rich cluster and to date only 231 redshift have been obtained for cluster members. A larger sample is needed to categorically rule out the presence of substructure.

Several authors have claimed that A3667 is observed in a post-merger state (Knopp et al. 1996; Markevitch et al. 1999; Roettiger et al. 1999; Röttgering et al. 1997; Vikhlinin et al. 2001). This may be consistent with the high velocity dispersion and mixing in the cluster. If the observed diffuse radio emission was generated via a cluster-cluster merger it is expected that the collisional velocities would be a few thousand $\mathrm{km} \mathrm{s}^{-1}$. If such a merger occurred, the current data suggest that the merger axis must be very close to the plane of the sky. This would partly account for the lack of observable subgroup peaks in the velocity data.

Full understanding of the the dynamical situation in A3667 will require multi-wavelength analysis. We have obtained detailed new radio data for the entire cluster at 1.4 , and $2.4 \mathrm{GHz}$; we will use this in conjunction with previously published radio ( $843 \mathrm{MHz}$ ) and $\mathrm{X}$-ray data to further investigate this cluster. Findings will be published in a future paper.

Acknowledgements. We gratefully acknowledge the 2dFGRS team for use of the 2dfdr pipeline code, and especially Dr Terry Bridges and Dr Russell Cannon for help with the observations and reduction. We thank Dr Keith Ashman for use of his mixture model analysis code, Dr Christopher Hollitt for help with the Matlab code, and Dr Jim Caswell and Dr Michiel van Haarlem for constructive comments on the manuscript. We thank the referee for the suggestion of several statistical tests and finally we thank the editor John Beckman for his extreme patience in dealing with the lengthy delays associated with this manuscript.

\section{References}

Abell, G. O., Corwin, H. G., \& Olowin, R. P. 1989, ApJS, 70, 1 Ashman, K. A., Bird, C. M., \& Zepf, S. E. 1994, AJ, 108, 2348 Bailey, J., Glazebrook, K., \& Bridges, T. 2002, 2dF Users Manual version 2.0 Bartholomew, L. J., Rose, J. A., Gaba, A. E., \& Caldwell, N. 2001, AJ, 122, 2913

Belloni, P., Bruzual, A. G., Thimm, G. J., \& Roser, H.-J. 1995, A\&A, 297, 61 Belsole, E., Sauvageot, J. L., Pratt, G. W., \& Bourdin, H. 2005, A\&A, 430, 385 Blake, C., Pracy, M. B., Couch, W. J., et al. 2004, MNRAS, 355, 713

Burns, J. O., Loken, C., Roettiger, K., et al. 2002, New Astron. Rev., 46, 135 Caldwell, N., \& Rose, J. A. 1997, AJ, 113, 492

Clarke, T. E., \& Ensslin, T. 2006, Astron. Nachr., 327, 553

Colless, M. 1998, in Looking Deep in the Southern Sky, ed. R. Morganti, \& W. J. Couch, 9

Colless, M., Dalton, G., Maddox, S., et al. 2001, MNRAS, 328, 1039

Couch, W. J., \& Sharples, R. M. 1987, MNRAS, 229, 423

Dressler, A., \& Shectman, S. A. 1988, AJ, 95, 985

Durret, F., Lima Neto, G. B., \& Forman, W. 2005, A\&A, 432, 809

Ebeling, H., Voges, W., Böhringer, H., et al. 1996, MNRAS, 283, 1103

Feretti, L. 2004, ArXiv Astrophysics e-prints

Ferrari, C., Hunstead, R. W., Feretti, L., Maurogordato, S., \& Schindler, S. 2006, A\&A, 457, 21

Fitchett, M. 1988, MNRAS, 230, 161

Fitchett, M., \& Webster, R. 1987, ApJ, 317, 653

Galaz, G. 2000, AJ, 119, 2118

Giovannini, G., \& Feretti, L. 2002, Highlights of Astronomy, 12, 513

Girardi, M., Fadda, D., Giuricin, G., et al. 1996, ApJ, 457, 61

Girardi, M., Giuricin, G., Mardirossian, F., Mezzetti, M., \& Boschin, W. 1998, ApJ, 505, 74

Govoni, F., Markevitch, M., Vikhlinin, A., et al. 2004, ApJ, 605, 695

Harris, D. E., Kapahi, V. K., \& Ekers, R. D. 1980, A\&AS, 39, 215

Henry, J. P., Finoguenov, A., \& Briel, U. G. 2004, ApJ, 615, 181

Hogg, D. W., Masjedi, M., Berlind, A. A., et al. 2006, ApJ, 650, 763

Hudson, D. S., Henriksen, M. J., \& Colafrancesco, S. 2004, in The Riddle of Cooling Flows in Galaxies and Clusters of galaxies, ed. T. Reiprich, J. Kempner, \& N. Soker, 47

Johnston-Hollitt, M. 2003, Ph.D. Thesis

Knopp, G. P., Henry, J. P., \& Briel, U. G. 1996, ApJ, 472, 125

Kuo, P.-H., Hwang, C.-Y., \& Ip, W.-H. 2004, ApJ, 604, 108

Markevitch, M., Sarazin, C. L., \& Vikhlinin, A. 1999, ApJ, 521, 526

Melnick, J., \& Quintana, H. 1981, AJ, 86, 1567

Miniati, F., Jones, T. W., Kang, H., \& Ryu, D. 2001, ApJ, 562, 233

Peacock, J. A. 1983, MNRAS, 202, 615

Pfrommer, C., Ensslin, T. A., \& Springel, V. 2007, ArXiv e-prints, 707

Poggianti, B. M. 2003, Ap\&SS, 285, 121

Pratt, G. W., Böhringer, H., Croston, J. H., et al. 2007, A\&A, 461, 71

Proust, D., Mazure, A., Sodré, L., Capelato, H., \& Lund, G. 1988, A\&AS, 72, 415

Roettiger, K., Burns, J. O., \& Stone, J. M. 1999, ApJ, 518, 603

Rose, J. A., Gaba, A. E., Christiansen, W. A., et al. 2002, AJ, 123, 1216

Röttgering, H. J. A., Wieringa, M. H., Hunstead, R. W., \& Ekers, R. D. 1997, MNRAS, 290, 577

Schuecker, P., \& Böhringer, H. 1999, in Diffuse Thermal and Relativistic Plasma in Galaxy Clusters, 43

Sodré, L. J., Capelato, H. V., Steiner, J. E., Proust, D., \& Mazure, A. 1992, MNRAS, 259, 233

Tonry, J., \& Davis, M. 1979, AJ, 84, 1511

Tran, K.-V. H., Franx, M., Illingworth, G., Kelson, D. D., \& van Dokkum, P. 2003, ApJ, 599, 865

Tran, K.-V. H., Franx, M., Illingworth, G. D., et al. 2007, ApJ, 661, 750

Tribble, P. C. 1993, MNRAS, 263, 31

Vikhlinin, A., Markevitch, M., \& Murray, S. S. 2001, ApJ, 551, 160

Vikhlinin, A. A., \& Markevitch, M. L. 2002, Astron. Lett., 28, 495

Whitmore, B. C., Zhang, Q., Leitherer, C., et al. 1999, AJ, 118, 1551

Xue, Y.-J., \& Wu, X.-P. 2000, ApJ, 538, 65 